\title{
The importance of the Ising model
}

\author{
Barry M. McCoy ${ }^{1}$ and Jean-Marie Maillard ${ }^{2}$
}

\author{
1. State University of New York, Stony Brook, NY \\ 2. University Paris 6, UMR 7600 CNRS, Paris, France
}

\begin{abstract}
Understanding the relationship which integrable (solvable) models, all of which possess very special symmetry properties, have with the generic nonintegrable models that are used to describe real experiments, which do not have the symmetry properties, is one of the most fundamental open questions in both statistical mechanics and quantum field theory. The importance of the two-dimensional Ising model in a magnetic field is that it is the simplest system where this relationship may be concretely studied. We here review the advances made in this study, and concentrate on the magnetic susceptibility which has revealed an unexpected natural boundary phenomenon. When this is combined with the Fermionic representations of conformal characters, it is suggested that the scaling theory, which smoothly connects the lattice with the correlation length scale, may be incomplete for $H \neq 0$.
\end{abstract}

\section{Introduction}

It may be rightly said that the two dimensional Ising model for $H=0$ is one of the most important systems studied in theoretical physics. It is the first statistical mechanical system which can be exactly solved which exhibits a phase transition. From the exact results for the free energy [1], spontaneous magnetization [2], 3] and correlation functions [4]-8] a point of view has been developed, which embraces the concepts of scaling, universality and conformal field theory, that extends the exact results of the Ising model to more general situations. These concepts are widely used to analyze both experiments and models of critical phenomena. Furthermore the correlation functions provide very concrete realizations of the concepts of mass and wave function renormalization used to define Euclidean quantum field theories.

However, starting with the work of Nickel 9, , 10, on the magnetic susceptibility new properties of the Ising model have been uncovered [1]-27] which go beyond what has been seen in the computations of the free energy, spontaneous magnetization and correlation functions. These new features need to be explored to see if there is relevant physics which is not incorporated in our current view of critical phenomena. In this article we will review these new phenomena and the relation they have with scaling theory and Euclidean quantum field theory.

In section 2 we define what will be meant by an Ising model. In section 3 
we review the known exact results for $H=0$. In addition to the well known results for the free energy [1] and the magnetization [2], 3] we will put particular emphasis on the magnetic susceptibility which has an expansion analogous [6, ,9, 10, to a Feynman diagram expansion. These Ising model integrals share with Feynman diagram integrals the property that the integrands are algebraic functions. It was shown, long ago, by Kashiwara and Kawai 28, that these Feynman integrals are holonomic (i.e. they satisfy overdetermined systems of partial differential equations). However, the infinite sum of diagrams will not have this property 1. These problems of power series expansions, where the infinite sum has different analytic properties than the coefficients of any finite power of the expansion parameter, are seen in the magnetic susceptibility of the Ising model 29. When expanded about $T=T_{c}$, this expansion of the susceptibility is not convergent but is only asymptotic [11] 26]. This feature also occurs in Quantum Electrodynamics [30] and Quantum Chromodynamics [31.

In section 4 we extend our considerations to $H \neq 0$, where much less is known and there is much to be learned. We will present perturbative studies, for $H$ near zero, of the two-point function [32, and the two-particle scattering amplitude [33. We will also present integrable perturbations of conformal field theory [34]-[36] about $T=T_{c}, H \neq 0$, about the Lee-Yang edge [37], 38], and the relation to Rogers-Ramanujan identities [39- 43$]$. We will finally discuss scaling theory [11, [26], 44], 45], and the study of the general non integrable perturbation of [46.

We conclude, in section 5, with an answer to the question of "Why is the Ising model is important?"

\section{What is the Ising model?}

We begin by defining what we mean by the two-dimensional Ising model in a magnetic field.

The simplest, and most well known, case is for nearest neighbor interactions on a square lattice defined by the classical interaction energy

$$
\mathcal{E}_{I}(H)=-\sum_{j=-L^{v}}^{L^{v}} \sum_{k=-L^{h}}^{L^{h}}\left\{E^{v} \cdot \sigma_{j, k} \sigma_{j+1, k}+E^{h} \cdot \sigma_{j, k} \sigma_{j, k+1}+H \cdot \sigma_{j, k}\right\},
$$

where $\sigma_{j, k}= \pm 1$ specify the "spin" at row $j$ and column $k$ of a square lattice. This definition can be extended to nearest neighbor interactions on other lattices by a straightforward expansion of the notation. We will impose either periodic, or cylindrical, boundary conditions.

For this interaction energy we are interested in computing the partition function

$$
Z_{L^{v}, L^{h}}(T, H)=\sum_{\sigma= \pm} \exp \left(-\mathcal{E}_{I}(H) / k_{B} T\right),
$$

\footnotetext{
${ }^{1}$ It has first remarked by Enting and Guttmann 29], on the anisotropic lattice, that the susceptibility of the square Ising model is not D-finite [29. The isotropic susceptibility is also non-holonomic (but this is not a consequence of the previous anisotropic result).
} 
where $k_{B}$ is the Boltzmann constant and the sum is over all values of the spins $\sigma_{j, k}$.

From the partition function (2) one gets the free energy $F(T, H)$ in the thermodynamic limit

$$
-F(T, H) / k_{B} T=\lim _{L^{v}, L^{h} \rightarrow \infty} \frac{1}{L^{v} L^{h}} \ln Z_{L^{v}, L^{h}}(T, H),
$$

the magnetization

$$
\mathcal{M}(T, H)=-\frac{\partial}{\partial H} F(T, H)=\left\langle\sigma_{0,0}\right\rangle,
$$

the magnetic susceptibility

$$
\chi(T, H)=\frac{\partial M(T, H)}{\partial H}=\frac{1}{k_{B} T} \sum_{M=-\infty}^{\infty} \sum_{N=-\infty}^{\infty}\left\{\left\langle\sigma_{0,0} \sigma_{M, N}\right\rangle-\mathcal{M}^{2}(T, H)\right\},
$$

and the internal energy

$$
u=k_{B} T^{2} \frac{\partial}{\partial T} F(T, H) / k_{B} T,
$$

where we have used the definition of the thermal average of an operator $O$

$$
\langle O\rangle=\lim _{L^{v}, L^{h} \rightarrow \infty} Z_{L^{v}, L^{h}}^{-1}(T, H) \cdot \sum_{\sigma= \pm} O \exp \left(-\mathcal{E}_{I}(H) / k_{B} T\right) .
$$

We note, in particular, that the magnetic susceptibility, at $H=0$, is written, in terms of the two-point function, as

$$
k_{B} T \cdot \chi(T, 0)=\sum_{M=-\infty}^{\infty} \sum_{N=-\infty}^{\infty}\left\{\left\langle\sigma_{0,0} \sigma_{M, N}\right\rangle-\mathcal{M}^{2}\right\}
$$

where

$$
\mathcal{M}=\lim _{H \rightarrow 0+} M(T, H),
$$

is the spontaneous magnetization. For the nearest neighbor interaction (11), the internal energy (6) reads:

$$
u=-E^{v} \cdot\left\langle\sigma_{0,0} \sigma_{1,0}\right\rangle-E^{h} \cdot\left\langle\sigma_{0,0} \sigma_{0,1}\right\rangle-H \cdot\left\langle\sigma_{0,0}\right\rangle .
$$

The interaction energy of nearest neighbor Ising model (1) may be generalized to interactions farther than nearest neighbors with interaction energy:

$$
\begin{aligned}
\mathcal{E}_{G}=- & \sum_{j=-L^{v}}^{L^{v}} \sum_{k=-L^{h}}^{L^{h}} \sum_{j^{\prime}=-L^{v}}^{L^{v}} \sum_{k^{\prime}=-L^{h}}^{L^{h}} E\left(\left|j-j^{\prime}\right|,\left|k-k^{\prime}\right|\right) \cdot \sigma_{j, k} \sigma_{j^{\prime}, k^{\prime}} \\
& -H \cdot \sum_{j=-L^{v}} \sum_{k=-L^{h}}^{L^{v}} \sigma_{j, k} .
\end{aligned}
$$


This reduces to (1) when $E(1,0)=E^{v} / 2, E(0,1)=E^{h} / 2$ and all other $E(j, k)=0$. The free energy, magnetization and susceptibility are all computed by replacing $\mathcal{E}_{I}$ in (2) by $\mathcal{E}_{G}$. The internal energy (10) generalizes to

$$
u=-\sum_{j=-L^{v}}^{L^{v}} \sum_{k=-L^{h}}^{L^{h}} E(|j|,|k|) \cdot\left\langle\sigma_{0,0} \sigma_{j, k}\right\rangle-H \cdot\left\langle\sigma_{0,0}\right\rangle .
$$

\section{What do we know for $H=0$ ?}

We have obtained more exact results for the nearest neighbor Ising model at $H=0$ than for any other system in statistical mechanics.

\subsection{Free energy}

The free energy of the nearest neighbor Ising model with $H=0$ was computed by Onsager [1] in 1944

$-F / k_{B} T=\ln 2$

$+\frac{1}{8 \pi^{2}} \int_{0}^{2 \pi} d \theta_{1} \int_{0}^{2 \pi} d \theta_{2} \ln \left[\cosh \frac{2 E^{h}}{k_{B} T} \cosh \frac{2 E^{v}}{k_{B} T}-\sinh \frac{2 E^{h}}{k_{B} T} \cos \theta_{1}-\sinh \frac{2 E^{v}}{k_{B} T} \cos \theta_{2}\right]$.

This free energy has a singularity at $T=T_{c}$ determined by

$$
\sinh 2 E^{v} / k_{B} T_{c} \cdot \sinh 2 E^{h} / k_{B} T_{c}=1 .
$$

This temperature $T_{c}$ is referred to as the critical temperature. The free energy may be expanded, about $T=T_{c}$, as

$$
-F / k_{B} T=\left(T-T_{c}\right)^{2} \cdot \ln \left|T-T_{c}\right| \cdot F_{1}\left(T-T_{c}\right)+F_{2}\left(T-T_{c}\right),
$$

where $F_{1}$, and $F_{2}$, are analytic at $T=T_{c}$ (i.e. they both have convergent power series expansions).

\subsection{Spontaneous magnetization}

The spontaneous magnetization was first announced by Onsager [2] in 1949, and a proof was given by Yang $[\underline{3}$ in 1952

$$
\mathcal{M}=\left(1-k^{2}\right)^{1 / 8}
$$

where

$$
k=\left(\sinh 2 E^{v} / k_{B} T \cdot \sinh 2 E^{h} / k_{B} T\right)^{-1},
$$

for $T<T_{c}$, and is zero for $T>T_{c}$. The history of the Onsager result is given in a most interesting paper of Baxter [47]. 


\subsection{Correlation functions}

All correlation functions of the nearest neighbor Ising model at $H=0$ may be expressed as determinants. These are particularly simple for the row correlation $\left\langle\sigma_{0,0} \sigma_{0, N}\right\rangle$, and the diagonal correlations $\left\langle\sigma_{0,0} \sigma_{N, N}\right\rangle$, which are given by

$$
D_{N}=\left|\begin{array}{llll}
a_{0} & a_{-1} & \cdots & a_{-N+1} \\
a_{1} & a_{0} & \cdots & a_{-N+2} \\
\vdots & \vdots & & \vdots \\
a_{N-1} & a_{N-2} & \cdots & a_{0}
\end{array}\right|
$$

with

$$
a_{n}=\frac{1}{2 \pi} \int_{0}^{2 \pi} d \theta \cdot e^{-i n \theta} \cdot\left[\frac{\left(1-\alpha_{1} e^{i \theta}\right)\left(1-\alpha_{2} e^{-i \theta}\right)}{\left(1-\alpha_{1} e^{-i \theta}\right)\left(1-\alpha_{2} e^{i \theta}\right)}\right]^{1 / 2},
$$

where for $\left\langle\sigma_{0,0} \sigma_{N, N}\right\rangle$

$$
\alpha_{1}=0, \quad \alpha_{2}=\left(\sinh 2 E^{v} / k_{B} T \sinh 2 E^{h} / k_{B} T\right)^{-1},
$$

and for $\left\langle\sigma_{0,0} \sigma_{0, N}\right\rangle$

$$
\alpha_{1}=e^{-2 E^{v} / k_{B} T} \cdot \tanh E^{h} / k_{B} T, \quad \alpha_{2}=e^{-2 E^{v} / k_{B} T} \cdot \operatorname{coth} E^{h} / k_{B} T .
$$

From this determinantal representation (18) of $\left\langle\sigma_{0,0} \sigma_{0, N}\right\rangle$, and $\left\langle\sigma_{0,0} \sigma_{N, N}\right\rangle$, it is easy to obtain the behavior of the correlation function as $T \rightarrow T_{c}$. The integrals $a_{n}$ all have logarithmic singularities at $T=T_{c}$. and we find [11, [48] for both the row and the diagonal correlations that $D_{N}$ has an expansion of the form

$$
D_{N}=\sum_{p=0}^{N} \sum_{n=0}^{\infty} d^{(p, n)}\left(\ln \left|T-T_{c}\right|\right)^{p} \cdot\left|T-T_{c}\right|^{p^{2}+n},
$$

where for each $p$ the sum over $n$ converges (and thus defines an analytic function). The correlations $\left\langle\sigma_{0,0} \sigma_{M, N}\right\rangle$ have a similar expansion in terms of powers of $\ln \left|T-T_{c}\right|$ multiplied by functions which are analytic at $T=T_{c}$.

When $T=T_{c}$ the determinant for the diagonal correlation reduces to a Cauchy determinant. It is explicitly evaluated as [5], yielding

$$
\left\langle\sigma_{0,0} \sigma_{N, N}\right\rangle=\left(\frac{2}{\pi}\right)^{N} \cdot \prod_{j=1}^{N-1}\left(1-\frac{1}{4 j^{2}}\right)^{l-N} .
$$

From this, we find as $N \rightarrow \infty$ that

$$
\left\langle\sigma_{0,0} \sigma_{N, N}\right\rangle=\frac{A_{c}}{N^{1 / 4}} \cdot\left(1-\frac{1}{64 N^{2}}+O\left(N^{-4}\right)\right),
$$

with

$$
A_{c}=2^{1 / 12} \cdot \exp \left[3 \zeta^{\prime}(-1)\right] \sim 0.6450 \cdots
$$

where $\zeta^{\prime}(z)$ is the derivative of the zeta function.

However, for $T \neq T_{c}$, the representation (18) is not an efficient way to study the limit $M, N \rightarrow \infty$. 


\subsection{Form factor expansions}

To study the correlation functions $\left\langle\sigma_{0,0} \sigma_{M, N}\right\rangle$ when $M, N \rightarrow \infty$, the determinants are re-expressed in an "exponential form" [6] for $T<T_{c}$ as

$$
\left\langle\sigma_{0,0} \sigma_{M, N}\right\rangle=\mathcal{M}^{2} \cdot \exp \sum_{n=1}^{\infty} F_{-}^{(n)}(T ; M, N)
$$

and, for $T>T_{c}$

$$
\left\langle\sigma_{0,0} \sigma_{M, N}\right\rangle=\mathcal{M}_{+}^{2} \cdot \sum_{j=1}^{\infty} G^{(2 j-1)}(T ; M, N) \cdot \exp \sum_{n=1}^{\infty} F_{+}^{(2 n)}(T ; M, N),
$$

where

$$
\mathcal{M}_{+}=\left[1-\left(\sinh 2 E^{v} / k_{B} T \cdot \sinh 2 E^{h} / k_{B} T\right)^{2}\right]^{1 / 8},
$$

is referred to as the disorder parameter and is the value of the spontaneous magnetization on the dual lattice where $E^{v}$ and $E^{h}$ are replaced by $E^{* h}$ and $E^{* v}$ defined as

$$
\sinh 2 E^{* v} / k_{B} T=1 / \sinh 2 E^{v} / k_{B} T, \quad \sinh 2 E^{* h} / k_{B} T=1 / \sinh 2 E^{h} / k_{B} T .
$$

The functions $F_{ \pm}^{(n)}(T ; M, N)$ and $G^{(n)}(T ; M, N)$ are $n$-dimensional integrals. These exponentials can be expanded, and terms combined 6], 9], 10] into what is referred to as the form factor expansion. For $T<T_{c}$ this expansion is

$$
\left\langle\sigma_{0,0} \sigma_{M, N}\right\rangle=(1-t)^{1 / 4} \cdot\left\{1+\sum_{n=1}^{\infty} f_{M, N}^{(2 n)}(T)\right\},
$$

with $f_{M, N}^{(2 n)}(T)$ a $2 n$-dimensional integral and:

$$
t=\left(\sinh 2 E^{v} / k_{B} T \cdot \sinh 2 E^{h} / k_{B} T\right)^{-2} .
$$

For $T>T_{c}$ the form factor expansion reads

$$
\left\langle\sigma_{0,0} \sigma_{M, N}\right\rangle=(1-t)^{1 / 4} \cdot \sum_{n=0}^{\infty} f_{M, N}^{(2 n+1)}(T),
$$

where $f_{M, N}^{(2 n+1)}(T)$ is a $2 n+1$ dimensional integral, and

$$
t=\left(\sinh 2 E^{v} / k_{B} T \cdot \sinh 2 E^{h} / k_{B} T\right)^{2} .
$$

For the diagonal correlations $\left\langle\sigma_{0,0} \sigma_{N, N}\right\rangle$ a simpler alternative form of $f_{N, N}^{(n)}(t)$ is given in [17, and proven in [18.

The behavior of $\left\langle\sigma_{0,0} \sigma_{M, N}\right\rangle$ is easily obtained, from this form factor expansion when $T \neq T_{c}$, because $f_{M, N}^{(n)}(T)$ has an exponential decay

$$
f_{M, N}^{(n)}(T) \sim e^{-\kappa(T ; M / N) \cdot n \cdot R}
$$


where $R^{2}=M^{2}+N^{2}$, where $\kappa(T ; M / N)$ depends on the ratio $M / N$, and vanishes, when $T \rightarrow T_{c}$, as:

$$
\kappa(T ; M / N) \sim\left|T-T_{c}\right| .
$$

This exponential decay defines a second length scale which, when $T \rightarrow T_{c}$, is infinitely great compared to the lattice length scale which defines the interaction energy (11). It is worth noting that the form (34) implies that the angular dependence of the decay of the correlation functions is the same for $T>T_{c}$ and $T<T_{c}$.

However, as $T \rightarrow T_{c}$, each term of the form factor expansion vanishes because $(1-t)^{1 / 4}$ vanishes, and each $f_{M, N}^{(n)}(T)$ is finite at $T=T_{c}$. The singularities of the $f_{M, N}^{(n)}(T)$ are all at $T=T_{c}$. These functions satisfy Fuchsian linear differential equations 17, and have logarithmic singularities at $T=T_{c}$ where the highest power of $\ln \left|T-T_{c}\right|$ is $n$. It therefore requires a detailed infinite summation of powers of logarithms to reproduce the behavior (22) for fixed $M, N$ as $T \rightarrow T_{c}$. In fact, this type of demonstration has never been carried out.

\subsection{Differential equations for $\left\langle\sigma_{0,0} \sigma_{N, N}\right\rangle$}

The correlation functions $\left\langle\sigma_{0,0} \sigma_{M, N}\right\rangle$ are holonomic (D-finite): they satisfy a set of partial linear differential equations in the variables $\sinh E^{v, h} / k_{B} T$. This is exactly the holonomic property of Feynman integrals, shown by Kashiwara and Kawai [28. For the diagonal case the more specialized result holds that $\left\langle\sigma_{0,0} \sigma_{N, N}\right\rangle$ satisfies a linear Fuchsian equation. The order of this linear differential equation is $N+1$, which is the minimum order needed to accommodate the singular terms at $T=T_{c}, \ln ^{p}\left|T-T_{c}\right|$ with $0 \leq p \leq N$ of (22), which were directly obtained from the determinantal representation (18). A few examples are given in [16].

However the diagonal correlation $\left\langle\sigma_{0,0} \sigma_{N, N}\right\rangle$ has the much more remarkable property, discovered by Jimbo and Miwa 8 in 1980, that it satisfies a second order non-linear differential equation

$$
\begin{aligned}
& \left(t \cdot(t-1) \cdot \frac{d^{2} \sigma}{d t^{2}}\right)^{2}= \\
& \quad N^{2} \cdot\left((t-1) \cdot \frac{d \sigma}{d t}-\sigma\right)^{2}-4 \frac{d \sigma}{d t} \cdot\left((t-1) \frac{d \sigma}{d t}-\sigma-\frac{1}{4}\right) \cdot\left(t \frac{d \sigma}{d t}-\sigma\right) .
\end{aligned}
$$

For $T>T_{c}$ the diagonal correlation is related to $\sigma$ by

$$
\sigma(t)=t \cdot(t-1) \cdot \frac{d}{d t} \log \left\langle\sigma_{0,0} \sigma_{N, N}\right\rangle-\frac{1}{4},
$$

with the boundary condition at $t=0$

$$
\left\langle\sigma_{0,0} \sigma_{N, N}\right\rangle=t^{N / 2} \cdot \frac{(1 / 2)_{N}}{N !}+O\left(t^{1+N / 2}\right),
$$


and, for $T<T_{c}$, it is related to $\sigma$ by

$$
\sigma(t)=t \cdot(t-1) \cdot \frac{d}{d t} \log \left\langle\sigma_{0,0} \sigma_{N, N}\right\rangle-\frac{t}{4},
$$

with the boundary condition

$$
\left\langle\sigma_{0,0} \sigma_{N, N}\right\rangle=(1-t)^{1 / 4} \cdot\left\{1-\frac{t^{N+1}}{2 N+1}\left(\frac{(1 / 2)_{N+1}}{(N+1) !}\right)^{2}+O\left(t^{N+2}\right)\right\},
$$

where $(a)_{N}=a(a+1) \cdots(a+N-1)$ is Pochhammer's symbol $\left(N \geq 1,(a)_{0}=1\right)$. These boundary conditions are obtained from the leading terms of the form factor expansions as $t \rightarrow 0$. Equation (36) is an alternative form of the Painlevé VI equation 49, called the $\sigma$-form of Painlevé VI.

No nonlinear isomonodromic (Garnier [50, 51]) systems of partial differential equations have been derived for the general correlation function $\left\langle\sigma_{0,0} \sigma_{M, N}\right\rangle$, even though they almost certainly exist. Such isomonodromic systems would yield, by differential algebra elimination, in the isotropic case, higher nonlinear order differential equations with the Painlevé property. Some exact results for $\left\langle\sigma_{0,0} \sigma_{N, N-1}\right\rangle$ are actually given by Witte in [52.

However $\left\langle\sigma_{0,0} \sigma_{M, N}\right\rangle$ does satisfy quadratic difference equations [53, [54]

$$
\begin{aligned}
& \sinh 2 E^{h} / k_{B} T \cdot\left\{C(M, N)^{2}-C(M, N-1) C(M \cdot N+1)\right\} \\
& +\sinh 2 E^{* v} / k_{B} T \cdot\left\{C^{*}(M, N)^{2}-C^{*}(M-1, N) C^{*}(M+1, N)\right\}=0, \\
& \sinh 2 E^{v} / k_{B} T \cdot\left\{C(M, N)^{2}-C(M-1) C(M+1, N)\right\} \\
& +\sinh 2 E^{* h} / k_{B} T \cdot\left\{C^{*}(M, N)^{2}-C^{*}(M, N-1) C^{*}(M, N+1)\right\}=0,
\end{aligned}
$$

where

$$
C(M, N)=\left\langle\sigma_{0,0} \sigma_{M, N}\right\rangle,
$$

and where $C^{*}(M, N)$ are the correlations on the dual lattice (29). At $T=T_{c}$, where $\sinh 2 E^{* . i} / k_{B} T=\sinh 2 E^{i} / k_{B} T$ for $i=v, h$, and $C^{*}(M, N)=C(M, N)$, these equations reduce to the discrete imaginary time Hirota equation [55].

\subsection{Susceptibility}

By using the form factor expansions (30) and (32) in (51), we obtain the expansion for the susceptibility as the infinite sum of $n$ "particle" contributions

$$
\begin{array}{ll}
k_{B} T \cdot \chi_{+}(T)=(1-t)^{1 / 4} \cdot t^{-1 / 4} \cdot \sum_{j=0}^{\infty} \chi^{(2 j+1)}(T), & \text { for } T>T_{c} \\
k_{B} T \cdot \chi_{-}(T)=(1-t)^{1 / 4} \cdot \sum_{j=1}^{\infty} \chi^{(2 j)}(T), & \text { for } T<T_{c} .
\end{array}
$$

where explicit forms of the $\chi^{(j)}(T)$, as $j-1$ dimensional integrals for the general anisotropic lattice, are given in [11]. These series are convergent for $T \neq T_{c}$. 
We may also consider what we call the "diagonal susceptibility", defined as:

$$
k_{b} T \cdot \chi_{d}(t)=\sum_{N=-\infty}^{\infty}\left\{\left\langle\sigma_{0,0} \sigma_{N, N}\right\rangle-\mathcal{M}^{2}\right\} .
$$

By use of the form of the diagonal form factor expansion of [17]-[18] we obtain as analogues of (45) and (44)

$$
\begin{array}{ll}
k_{B} T \cdot \chi_{d+}(T)=(1-t)^{1 / 4} \cdot t^{-1 / 4} \cdot \sum_{j=0}^{\infty} \chi_{d}^{(2 j+1)}(t), & \text { for } T>T_{c}, \\
k_{B} T \cdot \chi_{d-}(T)=(1-t)^{1 / 4} \cdot \sum_{j=1}^{\infty} \chi_{d}^{(2 j)}(t), & \text { for } T<T_{c},
\end{array}
$$

where the $\chi_{d}^{(n)}(t)$ are given in [59]. These series are also convergent for $T \neq T_{c}$.

The series expansions (44) and (45) are, what we referred to in the introduction as, the analogue of a "Feynman diagram expansion".

The integrals for $\chi^{(n)}(T)$, and $\chi_{d}^{(n)}(T)$, have been extensively studied [12][25]. It is quite instructive to contrast them with the form factor integrals $f_{M, N}^{(n)}(T)$ from whence they came.

When $T \rightarrow T_{c}$ each $\chi^{(n)}(T)$ diverges as $\left|T-T_{c}\right|^{-2}$ with a coefficient which depends on $n$ and rapidly decreases as $n$ increases. Thus, in terms of the variable

$$
\tau=\frac{1}{2} \cdot\left(\sinh ^{-1} 2 E / k_{B} T-\sinh 2 E / k_{B} T\right),
$$

we have, for the isotropic lattice [6], [56, [11] as $\tau \rightarrow 0$

$$
k_{B} T \cdot \chi_{ \pm}(\tau) \longrightarrow \sqrt{2} \cdot C_{ \pm} \cdot|\tau|^{-7 / 4} .
$$

The constants $C_{-}$and $C_{+}$are different, and are given as infinite series

$$
C_{-}=\sum_{n=1}^{\infty} C^{(2 n)}, \quad C_{+}=\sum_{n=0}^{\infty} C^{(2 n+1)}
$$

where the $C^{(n)}$ are $C^{(n)}=2^{-n} \pi^{n-1} D_{n}$, with [57.

$$
D_{n}=\frac{4}{n !} \int_{0}^{\infty} \cdots \int_{0}^{\infty} \frac{d u_{1}}{u_{1}} \cdots \frac{d u_{n}}{u_{n}} \cdot \frac{\prod_{i<j}\left(\frac{u_{i}-u_{j}}{u_{i}+u_{j}}\right)^{2}}{\left(\sum_{j=1}^{n}\left(u_{j}+1 / u_{j}\right)\right)^{2}}
$$

These integrals have been studied for $n=1, \cdots, 6$. The first terms in (51) have been analytically evaluated in [6]:

$$
C^{(1)}=1, \quad C^{(2)}=\frac{1}{12 \pi} .
$$


The next leading term was analytically evaluated by Tracy [58] as

$$
C^{(3)}=\frac{1}{2 \pi^{2}} \cdot\left(\frac{\pi^{2}}{3}+2-3 \sqrt{3} \mathrm{Cl}_{2}(\pi / 3)\right)=8.1446 \cdots \times 10^{-4},
$$

where

$$
\mathrm{Cl}_{2}(\theta)=\sum_{n=1}^{\infty} \frac{\sin n \theta}{n^{2}}
$$

is Clausen's function. The next term read:

$$
C^{(4)}=\frac{1}{16 \pi^{3}} \cdot\left(\frac{4 \pi^{2}}{9}-\frac{1}{6}-\frac{7}{2} \zeta(3)\right)=2.5448 \cdots \times 10^{-5} .
$$

Accurate numerical evaluations have been made [57 for $C^{(5)}$ and $C^{(6)}$ :

$$
\begin{aligned}
& C^{(5)}=\frac{1}{2^{5} \pi^{4}} \times 0.0024846057 \cdots=7.9709118 \cdots \times 10^{-7}, \\
& C^{(6)}=\frac{1}{2^{6} \pi^{5}} \times 0.0004891422 \cdots=2.497501 \cdots \times 10^{-8} .
\end{aligned}
$$

A curious feature of these results is that the ratio $C_{+} / C_{-}$is found to be closely approximated by $12 \pi$, and the succeeding terms decrease by approximately three orders of magnitude. The study of the constants $C_{-}$and $C_{+}$has been continued by high precision numerical computations [11, and the most recent evaluation [26], in 2011, is to 104 places.

For the diagonal susceptibility each $\chi_{d}^{(n)}(t)$ diverges as $(1-t)^{-1}$. One finds [27, 59]

$$
k_{B} T \cdot \chi_{d+}(T) \quad \longrightarrow \quad \frac{\left(1-x^{2}\right)^{1 / 4}}{1-x} \cdot \sum_{n=0}^{\infty} C_{d+}^{(2 n+1)}
$$

where $C_{d+}^{(1)}=1$, and

$$
C_{d+}^{(3)}=\frac{1}{3}-\frac{5 \pi}{9 \Gamma^{2}(5 / 6) \Gamma^{2}(2 / 3)}-\frac{8 \pi}{\Gamma^{2}(1 / 6) \Gamma^{2}(1 / 3)}=0.016329 \cdots
$$

and

$$
k_{B} T \cdot \chi_{d-} \quad \longrightarrow \quad(1-t)^{-3 / 4} \cdot \sum_{n=1}^{\infty} C_{d-}^{(2 n)}
$$

where $C_{d-}^{(2)}=\frac{1}{4}$, and

$$
C_{d-}^{(4)}=\frac{1}{8} \cdot\left(1-\frac{1}{3 \pi^{2}}[64-16 I]\right),
$$

with:

$$
I=-2.2128121 \cdots
$$


In contrast to the form factors $f_{M, N}^{(n)}(T)$, whose only singular point is $T=T_{c}$ where $T_{c}$ is real, the $\chi^{(n)}(T)$ 's have many further singularities. The first set of these singularities was found, by Nickel [9, 10, to be, for the isotropic case $E^{v}=E^{h}=E$, located at

$$
\cosh ^{2} 2 E / k_{B} T-\sinh 2 E / k_{B} T \cdot(\cos (2 \pi j / n)+\cos (2 \pi l / n)=0,
$$

with $([x]$ is the integer part of $x) 0 \leq j, l \leq[n / 2], j=l=0$ excluded (for $n$ even $j+l=n / 2$ is also excluded). Equivalently (64) reads $\sinh 2 E / k_{B} T_{j, l}=$ $s_{j, l}=1 / 2 \cdot(\cos (2 \pi j / n)+\cos (2 \pi l / n)) \pm i / 2 \cdot\left[\left(4-(\cos (2 \pi j / n)+\cos (2 \pi l / n))^{2}\right]^{1 / 2}\right.$. These Nickel's singularities are clearly on the unit circle $|s|=1$, or $|k|=1$. Do note that this is no longer the case for the anisotropic model. In the anisotropic case Nickel's singularities for the anisotropic $\chi^{(n)}$ 's become (see (3.22) of [11]):

$$
\begin{aligned}
& \cosh ^{2} 2 E^{v} / k_{B} T \cdot \cosh ^{2} 2 E^{h} / k_{B} T \\
& -\left(\sinh 2 E^{v} / k_{B} T \cdot \cos (2 \pi j / n)+\sinh 2 E^{h} / k_{B} T \cdot \cos (2 \pi l / n)\right)=0,
\end{aligned}
$$

with $j, l=1,2, \cdots n$. These (complex) algebraic curves (65), in the two complex variables $\sinh 2 E^{h} / k_{B} T$, $\sinh 2 E^{v} / k_{B} T$, are actually singular loc 2 for the D-finite system of PDE's satisfied by the anisotropic $\chi^{(n)}$ 's. These algebraic curves accumulates with increasing values of $n$, in the same way Nickel's singularities (64) accumulate on the unit circle $|s|=1$, in a certain (real) submanifold $\mathcal{S}$ of the two complex variables $\sinh 2 E^{h} / k_{B} T, \sinh 2 E^{v} / k_{B} T$ (four real variables). However, this "singularity manifold" $\mathcal{S}$ is not a codimensionone (real) submanifold (like the unit circle $|s|=1$ in the $s$-complex plane), but actually a codimension zero submanifold, as can also be seen on various analyses of complex temperature zeros (see for instance 60]). Note that this "singularity manifold" becomes very "slim" near the (critical) algebraic curve $k=\sinh 2 E^{h} / k_{B} T \cdot \sinh 2 E^{v} / k_{B} T=1$ (see for instance figures 1, 2 and 3 near the real axis in [60]). The relation between the analyticity in the two complex variables $\sinh 2 E^{h} / k_{B} T$ and $\sinh 2 E^{v} / k_{B} T$, and the single complex variable $T$ with $E^{v}$ and $E^{h}$ real and fixed, will be discussed elsewhere.

Back to the isotropic model, introducing the variable

$$
w=\frac{1}{2 \cdot\left(\sinh 2 E / k_{B} T+\left(\sinh 2 E / k_{B} T\right)^{-1}\right)},
$$

the singularities (64) for $n=3,4,5,6$ are given in table 1 , where we note, when $w$ is real, that $\sinh 2 E / k_{B} T$ is real for $-1 / 4 \leq w \leq 1 / 4$, and is complex with $\left|\sinh 2 E / k_{B} T\right|=1$ for $|w|>1 / 4$. Following [10] we define $\epsilon$, the deviation from the singular temperatures $T_{j, l}^{(n)}$ determined by (64), as $s^{-1}=(1-\epsilon) \cdot s_{j, l}^{-1}$, for $T<T_{c}$, and $s=(1-\epsilon) \cdot s_{j, l}$, for $T>T_{c}$. Then, for $T<T_{c}$, the singularity in $\chi^{(2 n)}(T)$, at $T_{j, l}$, reads

$$
A_{j, l}^{(2 n)} \cdot \epsilon^{2 n^{2}-3 / 2} .
$$

${ }^{2}$ This result on the anisotropic Ising model has been obtained from a Landau singularity analysis, generalizing [19 20] (S. Boukraa, S. Hassani and J-M. Maillard, unpublished results). 
and, for $T>T_{c}$, the singularity in $\chi^{(2 n+1)}(T)$ reads

$$
A_{j, l}^{(2 n+1)} \cdot \epsilon^{2 n(n+1)-1} \cdot \frac{\ln \epsilon}{\pi} .
$$

The amplitude $A_{j, l}^{(N)}$ is given by 10

$$
\begin{aligned}
A_{j, l}^{(N)} & =\frac{\left(N i \sin \theta_{j, l}\right)^{\left(N^{2}-3\right) / 2}}{\left(\sin ^{2} \phi^{(j)} \cos \phi^{(l)}+\sin ^{2} \phi^{(l)} \cos \phi^{(j)}\right)^{\left(N^{2}-1\right) / 2}} \\
& \times \frac{\prod_{m=1}^{N-1}\left(m ! / 2^{m}\right)}{\pi^{(N-3) / 2)} \sqrt{N} \Gamma\left(\left(N^{2}-1\right) / 2\right)}
\end{aligned}
$$

with

$$
\phi^{(m)}=2 \pi m / N, \quad 2 \cos \theta_{j, l}=\cos \phi^{(j)}+\cos \phi^{(l)} .
$$

Table 1: The Nickel singularities (64) for the isotropic case $E^{v}=E^{h}$ of $\chi^{(n)}$ for $n=3,4,5,6$

\begin{tabular}{|l|l|}
\hline$n$ & $w$ \\
\hline 3 & $-1 / 2,1$ \\
4 & $\pm 1 / 2$ \\
5 & $-1, \frac{-1 \pm \sqrt{5}}{4}, \frac{3 \pm \sqrt{5}}{2}$ \\
6 & $\pm 1, \quad \pm 1 / 3$ \\
\hline
\end{tabular}

The Fuchsian linear differential equations [12]-25] which the (isotropic) $\chi^{(n)}(T)$ satisfy, have singularities which accumulate on the unit circle, but, also, inside, and outside, this unit circle $\left|\sinh 2 E / k_{B} T\right|=1$. However, the series expansion of these integrals $\chi^{(n)}(T)$ are actually analytic inside the unit circle $\left(\left|\sinh 2 E / k_{B} T\right|<1\right)$. The singularities of a linear ODE and the singularities of a particular series-solution of an ODE do not coincide.

The singularities of the Fuchsian ODE may be obtained, from the integrand of the integrals, by the same "Landau" analysis [19] used to obtain singularities of Feynman diagrams.

For the isotropic $\chi^{(3)}$, in addition to the unit circle singularities (64), there are additional singularities at

$$
w=\frac{-3 \pm i \sqrt{7}}{8}, \quad \text { where } \quad s, s^{-1}=\frac{-1 \pm i \sqrt{7}}{2}
$$

and they actually correspond to complex multiplication of elliptic curves, Heegner numbers, and complex fixed points of the Landen transformation [20].

The singularities of $\chi^{(n)}(w)$ are to be contrasted with the diagonal susceptibility $\chi_{d}^{(n)}(t)$ which only have [19] singularities on the unit circle $|t|=1$. 
For $T<T_{c}$ the singularities of $\chi_{d}^{(2 n)}(t)$ are at $t_{0}=e^{2 \pi i j / n}$, and are of the form:

$$
A_{d ; j}^{(2 n)} \cdot \epsilon^{2 n^{2}-1} \cdot \ln \epsilon .
$$

For $T>T_{c}$ the singularities in $\chi_{d}^{(2 n+1)}(t)$ are at $t_{0}=e^{2 \pi j /(n+1 / 2)}$, and are of the form

$$
A_{d ; j}^{(2 n+1)} \cdot \epsilon^{(n+1)^{2}-1 / 2}
$$

where the amplitudes $A_{d ; j}^{(N)}$ have yet to be determined.

The linear differential operator for $\chi^{(3)}$ rightdivides the one for $\chi^{(5)}$ (in a direct sum structure 21]). Consequently, all the singularities of the linear differential operator for $\chi^{(3)}$ are also singularities of the operator for $\chi^{(5)}$. It was, however, seen [21], by means of a Fast Fourier Transform analysis of the series expansion of the integral for $\chi^{(5)}$, as a power series in $t$, that some $\chi^{(3)}$ singularities are not present in $\chi^{(5)}$. This is to be contrasted with a very recent result 61 for the diagonal susceptibility $\chi_{d}^{(5)}$ which has found that the singularities of $\chi_{d}^{(3)}$ are present in $\chi_{d}^{(5)}$.

\subsection{Natural boundary for the isotropic model}

It is striking that the number of singularities (64) in $\chi^{(n)}(T)$ increases with $n$, and becomes dense in the limit $n \rightarrow \infty$. This feature led Nickel [9] to the conclusion that, unless cancellations occur, there will be a unit circle natural boundary in the susceptibility $\chi(T)$ in the complex $s$ plane. The fact that ref. 21. demonstrates that some singularities of the $\chi^{(3)}$ series are not singularities of the $\chi^{(5)}$ series, supports the no cancellation assumption. The existence of a natural boundary, in the complex temperature plane, is not contemplated in the scaling theory of critical phenomena. The literature on natural boundary is quite narrow 62, as well as the methods and tools to study the neighborhood of a natural boundary. Curious situations may occur, like, for instance, the "radial porosity" one encounters with Borel's examples of monogenic functions (see section 10.5 page 21 of 63 ).

There is much that is, as yet, not understood about the properties of this Ising natural boundary. In the vicinity of any point on $|s|=1$, such that $s \neq \pm 1$, the local spacing of the singularities, in $\chi^{(n)}$, is of the order $n^{-2}$. However, for $s= \pm 1$ the local spacing is only $n^{-1}$, and the dependence of the amplitude (69), for large $N$, is different in these two cases.

An initial analysis was made in [11, for $s \rightarrow 1$, based on keeping only the singularities closest to $\tau=0$. This analysis yielded an essential singularity of the form $e^{-C / \tau^{2}}$ where $C$ is a constant. However, there is more to a natural boundary than just an essential singularity, and further analysis will be required to fully assess the properties following from the singularities in $\chi^{(n)}$. In particular we note that, in general, the limiting value at a point on a natural boundary depends on the direction of the approach, and, as suggested in [11, an asymptotic expansion about $|s|=1, s \neq \pm 1$, may not exist. The existence of a unit circle natural boundary in the isotropic square Ising model, seriously 
questions most of the scaling arguments taken for granted on this model. For example it is desirable to explore the relation between the equality $\gamma_{+}=\gamma_{-}$ for the susceptibility exponent $\gamma$, above and below $T_{c}$, and the unit circle natural boundary which disconnect 3 the inside and the outside of the unit circle, $|k|<1$ and $|k|>1$.

\subsection{Series expansions}

There is a second way of studying the susceptibility which is distinct from (and in a way complementary to) the use of the form factor expansion. By use of either (in principle) the determinant representation or (in practice) by the more sophisticated approach of using the nonlinear difference equations (41), (42) with the explicit result for $\left\langle\sigma_{0,0} \sigma_{N, N}\right\rangle$ of (23), and for $\left\langle\sigma_{0,0} \sigma_{N, N-1}\right\rangle$ of [52], very long expansions about $T=0$, and $T=\infty$, can be obtained. For the isotropic lattice $E^{v}=E^{h}=E$ this has been done in [11] and [26], and series expansions with may hundreds of terms have been obtained, not only on the square, but also on the triangular and honeycomb lattices.

These long expansions have been analyzed in 11 and 26]. This analysis leads to the following expansion of the susceptibility as $T \rightarrow T_{c}$ which generalizes the leading diverging term given in (50)

$$
k_{B} T \cdot \chi_{ \pm}=C_{ \pm} \cdot|\tau|^{-7 / 4} \cdot F_{ \pm}(\tau)+B(\tau),
$$

where

$$
F_{ \pm}(\tau)=1+\sum_{n=1}^{\infty} f_{n \pm} \cdot \tau^{n}
$$

and

$$
B(\tau)=\sum_{q=0}^{\infty} \sum_{p=0}^{[\sqrt{q}]} b^{(p, q)} \cdot\left(\ln \tau^{2}\right)^{p} \cdot \tau^{q},
$$

with $\tau$ taken to be real, and where the $b^{(p, q)}$ 's are the same for $T<T_{c}$ and $T>T_{c}$, and are functions depending on the lattice. Unlike the expansions (44), and (45), which converge we will see below that (76) is expected to only be asymptotic. The function $F_{ \pm}(\tau)$ is referred to, in [11] and [26], as a "scaling function".

It is greatly instructive to compare the result (74) of 11 and 26 with the behavior of the expansions (44) and (45), derived from the form factor expansion.

The term $B(\tau)$ is of the same form as the logarithmic terms (22) already seen in the determinantal form of the correlations. Such terms are present term by term in (5), and are referred to, in [11, as "short distance" contributions. However, in the expansion (44) and (45), the $\chi^{(n)}(T)$ have singularities, at $T \rightarrow T_{c}$, with powers of $\ln \left|T-T_{c}\right|$. Thus, term by term in (44) and (45), the

\footnotetext{
${ }^{3}$ As far as analysis of several complex variables 64 is concerned, the situation is even worse for the anisotropic Ising model, because of the codimension zero manifold $\mathcal{S}$ of section 3.6.
} 
coefficient $(1-t)^{1 / 4}$ is not cancelled out. Consequently there must be an infinite number of detailed cancellations to obtain the $B(\tau)$ of (76) from (44) and (45).

The most interesting question concerns the convergence of the infinite series in (75) and (76). This cannot, of course, be answered by a finite series, no matter how long and, in fact, the numerical results for $F_{ \pm}(\tau)$, and $b^{(p, q)}$ in [26], do not show divergent behavior with the number of terms which have been computed. However, the dense set of singularities in the $\chi^{(n)}(T)$, found by Nickel [9], 10] in analyzing the form factor integrals, must have a significant influence on this expansion. The influence of Nickel's singularities has been analyzed in [1] with the conclusion that there must be asymptotic behavior in, at least, some of the series in (75) and (76). It is argued in [11, from the results of (6.12), that the coefficients $b^{(p, 0)}$ form an asymptotic sequence for sufficiently large $p$. The behavior of $b^{(p, q)}$, for $p$ fixed and $q \rightarrow \infty$, and $f_{n \pm}$ as $n \rightarrow \infty$, remains to be carried out. The further effects caused by the Landau singularities [19] also remain to be studied.

\subsection{The scaling (field theory) limit at $H=0$}

One of the most important properties of the Ising model at $H=0$, as $T \rightarrow T_{c}$, is that it defines a Euclidean quantum field theory and gives a very concrete example of the concepts of mass and wave function renormalization.

We concretely illustrate this for the two point correlation $\left\langle\sigma_{0,0} \sigma_{M, N}\right\rangle$. For mass renormalization we define

$$
r=\kappa(T ; M / N) \cdot R,
$$

where $R^{2}=M^{2}+N^{2}$, and where $\kappa(T ; M / N)$ is the inverse correlation length introduced in subsection (3.4). We define what we call the scaling limit, for $T$ real, as

$$
T \longrightarrow T_{c}, \quad R \longrightarrow \infty,
$$

with $r$ fixed, and we recall from (35) that $\kappa$ vanishes as $T \rightarrow T_{c}$

$$
\kappa(T ; M / N) \sim A_{\kappa} \cdot(1-t) .
$$

For wave function renormalization we divide the correlation function by the factor $(1-t)^{1 / 4}$ which vanishes at $T=T_{c}$. When $T<T_{c}$ this factor is the square of the spontaneous magnetization. The interpretation is that the spins, which on that lattice have the values $\sigma= \pm 1$, are regarded as having a "natural size" of $\mathcal{M}$ in the scaling limit. Similarly, for $T>T_{c}$, the value of the disorder parameter $M_{+}$is interpreted as the natural size for $\sigma$.

We then define for $T \rightarrow T_{c} \pm$

$$
G_{ \pm}(r)=\lim _{\text {scaling }}(1-t)^{-1 / 4} \cdot\left\langle\sigma_{0,0} \sigma_{M, N}\right\rangle .
$$

By use of the form factor expansion we see that this limit exists. In the isotropic case this function is rotationally invariant. In the anisotropic case it becomes 
rotationally invariant if one uses the length variable $r$ (fixed)

$$
\left[\left(\frac{\sinh 2 E^{h} / k_{B} T_{c}}{\sinh 2 E^{v} / k_{B} T_{c}}\right)^{1 / 2} \cdot M^{2}+\left(\frac{\sinh 2 E^{v} / k_{B} T_{c}}{\sinh 2 E^{h} / k_{B} T_{c}}\right)^{1 / 2} \cdot N^{2}\right]^{1 / 2}(1-t)=r .
$$
kind

This function $G_{ \pm}(r)$ is expressed in terms of a Painlevé equation of the third

$$
\frac{d^{2} \eta}{d \theta^{2}}=\frac{1}{\eta}\left(\frac{d \eta}{d \theta}\right)^{2}-\frac{1}{\theta} \frac{d \eta}{d \theta}+\eta^{3}-\eta^{-1}
$$

as

$$
G_{ \pm}(r)=\frac{1 \mp \eta(r / 2)}{2 \eta(r / 2)^{1 / 2}} \cdot \exp \frac{1}{4} \int_{r / 2}^{\infty} d \theta \cdot \theta \cdot \frac{\left(1-\eta^{2}\right)^{2}-\left(\eta^{\prime}\right)^{2}}{\eta^{2}} .
$$

The second order equation (82) admits a one parameter family of solutions which decay exponentially, when $\theta \rightarrow \infty$, as

$$
\eta(\theta) \sim 1-\frac{2 \lambda}{\pi} \cdot K_{0}(2 \theta)
$$

where $K_{0}(2 \theta)$ is the modified Bessel function. The specific value $\lambda=1$ corresponds to the Ising model. The result was first announced in [56] and [65]. Two different proofs were originally given. The first, in [6], is based on Myers' work 66, on the scattering of electromagnetic radiation from a strip, and the second [7] is based on a direct manipulation of the exponential representation in the scaling limit.

An alternative derivation of the scaled two-point function was subsequently obtained [8] by directly taking the scaling limit of the Painlevé VI equation (36). This scaling leads to representation in terms of a Painlevé V function. The equivalence of this representation with the original Painlevé III form was shown in 67.

The scaling limit as defined by (77) and (78), which is used to define the scaled Green's function (80), is defined from the massive regime where the correlation on the lattice decays exponentially. It remains to connect this regime with the algebraic decay of the lattice correlations, at $T=T_{c}$, given by (24). We do this by extending the strict limiting definition (80) with the less precise statement that

$$
\left\langle\sigma_{0,0} \sigma_{M, N}\right\rangle \sim(1-t)^{1 / 4} \cdot G_{ \pm}(\kappa R),
$$

and examine (85) as $T \rightarrow T_{c}$, i.e. $t \rightarrow 1$ and $\kappa \rightarrow \infty$. Thus, we see that if, as $r \rightarrow 0$, we have

$$
G_{ \pm}(r) \quad \longrightarrow \quad A_{G} / r^{1 / 4}
$$

then (85) reduces to

$$
\left\langle\sigma_{0,0} \sigma_{M, N}\right\rangle \quad \longrightarrow \quad A_{G} \cdot\left(\frac{A_{\kappa}}{R}\right)^{1 / 4} .
$$


The exponent $1 / 4$ in (86) is shown in [7] to follow, from the local behavior of the Painlevé III equation at $\theta \rightarrow 0$, if the exponentially decaying boundary condition (84), at infinity, holds with $\lambda=1$.

The constant $A_{G}$ does not follow from a local property of the Painlevé function. and the limit of $G_{ \pm}(r)$, as $r \rightarrow 0$, will agree with $\left\langle\sigma_{0,0} \sigma_{M, N}\right\rangle$, as $R \rightarrow \infty$, if in addition to the functional form (86), it can be shown that

$$
A_{G} \cdot A_{\kappa}^{1 / 4}=A_{c},
$$

with $A_{c}$ given by (25). This crucial identity was first proven by Tracy 68, in 1991, by use of the scaling limit of the exponential forms of the correlation (26) and (27). It is perhaps worth pointing out that a derivation of the constant $A_{G}$ of (86) has never been directly obtained from the Painlevé III form (82), (883).

\section{The Ising model for $H \neq 0$}

The properties of the Ising model at $H=0$, presented in the previous section, are all found by exact computations which start with the definition (11) of the nearest neighbor Ising model and are mathematically rigorous. However when we extend our considerations to $H \neq 0$, this is not the case, and, with only a few exceptions, the results require some arguments which, while often extremely plausible, in fact include assumptions which remain to be proven. Nevertheless the work of the last 50 years has produced remarkable results which give a compelling scenario of the behaviour of the Ising model for $H \neq 0$. In this section will here discuss the following major contributions:

1. Perturbation for small $H$ for the two-point function 32 and two-body scattering for $T>T_{c}$ 33,

2. Conformal field theory 34 ,

3. Integrable perturbations of conformal field theory [35, 36,

4. The connection of integrable perturbations with Rogers-Ramanujan identities [39]- 43],

5. Scaling theory with irrelevant operators and nonlinear scaling fields [11, [26], 44], 45],

6. Non-integrable perturbations of conformal field theory [46].

\subsection{Perturbation for $H \sim 0$}

For $T<T_{c}$ the two point function has been studied perturbatively [32] for small $H$. It was found that if in the limit $H \rightarrow 0$ and $T \rightarrow T_{c-}$ from the low temperature side with the "scaled magnetic field $h$ fixed

$$
h=\lim _{\text {scaling }} \frac{H}{\left|T-T_{c}\right|^{15 / 8}},
$$


then the connected part of the two-point function, for $h \sim 0$ and large $r$, is

$$
G^{c}(r ; h) \sim \pi^{1 / 2} \cdot r^{-1 / 2} \cdot e^{-2 r} \cdot \sum_{j} a_{j}(h) \cdot e^{-r \kappa_{j}(h)}
$$

with

$$
\kappa_{j}(h)=h^{2 / 3} \cdot \lambda_{j} \quad \text { and } \quad a_{j}(h)=h \cdot a,
$$

where $a$ is a constant, and the $\lambda_{j}$ 's are solutions of

$$
J_{1 / 3}\left(\lambda^{3 / 2} / 3\right)+J_{-1 / 3}\left(\lambda^{2 / 3} / 3\right)=0,
$$

where $J_{n}(z)$ is the Bessel function of order $n$.

This perturbation is, in fact, a "singular" perturbation which is subject to some interpretation. In particular one is not able to distinguish between $r^{-1 / 2} e^{-\kappa r}$ and $K_{0}(\kappa r)$, where $K_{0}(z)$ is the modified Bessel function. If we make this replacement in (90), then the Fourier transform consists of a set of poles, and this result can be interpreted as an example of confinement of two "domain wall excitations" which interact by means of an infinitely weak linear confining potential, to produce a spectrum of "mesons". For $T>T_{c}$ the only effect on the leading behavior of the two-point function, for $h \sim 0$, is to shift the correlations length by a term proportional to $h^{2}$. From these computations a scenario is conjectured in 32 that, as we go from $T<T_{c}$ to $T>T_{c}$ in a path in the $(H, T)$ plane in the scaling limit, the Fourier transform of the two-point function will contain poles. These poles can be interpreted as quasi particles and the number of these poles will go, in a smooth fashion, from one at $T>T_{c}$, $H=0$, to the infinite number of poles for $T<T_{c}$ and $h \rightarrow 0$ given by (90).

Much more recently [33] the two-body scattering has been studied in the same small $h$ limit for $T>T_{c}$. One of the very significant results of this study is that, at large energies, the scattering is predominantly inelastic.

\subsection{Conformal field theory}

Conformal field theory is an entirely new approach to critical phenomana invented by Belavin, Polyakov and Zamolodchikov 34 in 1984. In this approach there is no lattice such as (1) and the theory is defined directly in the continuum. In particular, these continuum theories make no contact with the short distance terms, and the natural boundary, which were discussed in section 3 .

It is not our purpose here to present a survey of conformal field theory, which is well presented in the original paper 34] and, subsequently, in many places 69], 70. Instead, we will restrict our attention to a discussion of two integrable perturbations relevant to the Ising model, the $M(3,4)$ and the $M(2,5)$ minimal models.

The minimal model $M(3,4)$ describes the critical point of the Ising model at $T=T_{c}$ and $H=0$. It has two relevant operators for the energy and magnetization. The conformal dimension of energy is $1 / 2$, which means the two-point

function for energy is $r^{-2}$, and for magnetization is $1 / 16$, which means that the 
two-point spin-spin correlation is $r^{-1 / 4}$. These results agree which the exact results at $T=T_{c}$ of the Ising model (1).

There is, however, a second conformal field theory which is relevant to the Ising model, namely the $M(2,5)$ model which is related to the Lee-Yang edge.

In 1952 Lee and Yang [71 proved what is one of the few results exactly known for the Ising model in a magnetic field, namely that, for real interaction energies, the zeros of the partition function of an isotropic Ising model on a finite lattice, all lie on the circle $z=1$, where

$$
z=e^{-2 H / k_{B} T} \text {. }
$$

For $T>T_{c}$ these zeros are all bounded away from the unit circle $z=1$, and they pinch the real $z$-axis when $T \rightarrow T_{c}$. For $T<T_{c}$ they fill up the unit circle $|z|=1$. For $T>T_{c}$ the endpoint of the arc of zeros is called the Lee-Yang edge.

The confining of partition function zeros to an arc in the complex $z$ plane for real temperatures is to be contrasted with the zeros of the partition function in the complex plane $u=e^{-4 E / k_{B} T}$ for real $H$, where computer studies [72, on systems of size 4 up to $16 \times 16$ show that the zeroes for $H=0$ are located on curves only for very special boundary conditions [73], and for $H \neq 0$ there are regions of the complex $u$-plane where, even for these special boundary conditions, the zeros do not lie on curves.

The identification of the Lee-Yang edge as a critical point, with a continuum field theory interpretation, was first made by Fisher [74, and the identification of this field theory with the $M(2,5)$ minimal model was first made by Cardy 37. There is only one relevant operator and the dimension is $-1 / 5$, which means that the two-point function of this operator is $\left\langle\phi_{0} \phi_{r}\right\rangle$ is $r^{4 / 5}$. Because there is only one relevant operator, both the energy operator $\sigma_{j, k} \sigma_{j, k+1}$ and the spin operator $\sigma_{j, k}$ must have the same two-point function as the operator $\phi$ of the $M(2,5)$ model.

The value of the magnetic field $H=i H_{L Y}$, at the Lee-Yang edge, vanishes at $T \rightarrow T_{c}$ from above, as:

$$
H_{L Y}=C_{L Y} \cdot\left(T-T_{c}\right)^{15 / 8}
$$

Cardy has determined [37] that the density of zeros $\rho(\operatorname{Im}(H))$ diverges, when $\operatorname{Im}(H) \rightarrow H_{L Y}$, as:

$$
\rho(\operatorname{Im}(H)) \sim\left(\operatorname{Im}(H)-H_{L Y}\right)^{-1 / 6} .
$$

\subsection{Integrable perturbations of conformal field theory}

A perturbation of a conformal field theory is called integrable if it preserves an infinite number of the constants of the motion of the conformal field theory which is being perturbed. This concept was introduced by Zamolodchikov [35, [36] at a conference in 1988 , where he discovered that the perturbation of the $M(3,4)$

\footnotetext{
${ }^{4}$ We have also performed, with I. Jensen, calculations of zeros of partition function of the square Ising model in a magnetic field up to size $20 \times 30$.
} 
model, by both the energy and separately by the spin operator, have an infinite number of conservation laws. The $M(2,5)$ model was shown 38 to have an integrable perturbation by the operator $\phi$ in 1990 .

\subsubsection{The case of $M(3,4)$}

The perturbation of the minimal model $M(3,4)$ by the energy operator $\epsilon=$ $\sigma_{j, k} \sigma_{j, k+1}$ is, in fact, nothing more than saying that the original Ising model with nearest neighbor interactions (1) is integrable, which is manifestly true and completely not surprising. However, the discovery in [35, and [36, that the perturbation by the spin operator $\sigma_{j, k}$, which corresponds to the Ising model, at $T=T_{c}$ in a non zero magnetic field, is integrable came as a big surprise, because the lattice interaction (11), at the critical temperature with $H \neq 0$, is not integrable. Fortunately, this mystery was resolved in 1992 when an integrable lattice model was found [75] which does have the critical behaviour of the magnetization of $H^{1 / 15}$, found in 35 and 36 , for the perturbed conformal field theory. This is the behavior of the Ising magnetization obtained, decades ago, by simple scaling arguments for critical exponents.

The truly remarkable property of both, the perturbed conformal field theory and the lattice realization, is that they have an excitation spectrum with eight quasi-particles, and that the masses of these particles are proportional to the components of the Perron-Frobenius eigenvector of the Cartan matrix of the $E_{8}$ Lie algebra [35, [36. This is completely in accordance with the scenario proposed from the perturbative computation of 32 .

\subsubsection{The case of $M(2,5)$}

The integrable perturbation of the $M(2,5)$ model was investigated in [38. In this case there is only a single quasi particle excitation. A lattice realization is regime I of the hard hexagon model [76].

Because there is only one relevant operator the identification of this perturbation with the Lee-Yang edge, for real $T \neq T_{c}$ and complex magnetic field, should be equivalent to the corresponding edge in the complex energy (temperature) plan for real magnetic field $H \neq 0$. However, the complex energy partition zeros do not lie on curves the way the complex magnetic field zeros do. Thus, for this identification to hold, further restrictions on these complex energy zeros must hold in the vicinity of the edge. Consequently, the precise relation the energy edge has with the perturbed $M(2,5)$ model remains an open question.

\subsection{Rogers-Ramanujan identities}

All conformal field theories possess a Bose-Fermi duality 41, 42. The Bose form gives characters in the Roccha-Caridi form 77. by eliminating singular vectors from a Bosonic Fock space. For the minimal models $M\left(p, p^{\prime}\right)$, these 
characters are

$$
\chi_{r, s}^{\left(p, p^{\prime}\right)}=\frac{1}{(q)_{\infty}} \cdot \sum_{j=-\infty}^{\infty}\left(q^{j\left(p p^{\prime} j+r p^{\prime}-s p\right)}-q^{\left(p^{\prime} j+s\right)(p j+r)}\right),
$$

where

$$
(q)_{m}=\prod_{k=1}^{m}\left(1-q^{k}\right)
$$

for $1 \leq r \leq p-1$ and $1 \leq s \leq p^{\prime}-1$. This is a "field" representation of the theory, and can be thought of as being natural to characterize "short distance" (ultraviolet) properties. An equivalent representation is given in terms of exclusion statistics applied to a set of Fermionic quasi-particles. This gives rise to a Fermionic form of the characters. These Fermionic forms give a particle characterization of the spectrum, and can be thought of as the natural characterization of "long distance" (infrared) properties. The relation between the Bose and Fermi forms is a generalization of the famous Rogers-Ramanujan identities [78, [79].

The illustration of this duality for the Ising model, at $T=T_{c}, H=0$, as the $M(3,4)$ minimal model is very instructive.

From (2.8) of [39] the Fermionic representation of character $\chi_{1,2}^{(3,4)}$, for the spin operator of the minimal model $M(3,4)$ which characterizes the Ising conformal field theory, is

$$
\begin{aligned}
\chi_{1,2}^{(3,4)} & =\sum_{\substack{m=0 \\
m \text { odd }}}^{\infty} \frac{q^{m(m-1) / 2}}{(q)_{m}} \\
& =\sum_{\substack{m=0 \\
\text { even }}}^{\infty} \frac{q^{m(m-1) / 2}}{(q)_{m}} .
\end{aligned}
$$

Similar formulas hold for the identity character $\chi_{1,1}^{(3,4)}$, and the energy character $\chi_{2,1}^{(3,4)}$. These Fermionic forms match the particle excitations seen in the form factor expansion of the form factors for $H=0$. There is one type of excitation, and the index $m$ represents the contribution of a $m$-particle state. The sum over odd (even) $m$ in (98) (respectively (99)), corresponds to the odd (even) number of quasi-particles in the form factor expansion of the two-point function for $T>T_{c}\left(T<T_{c}\right)$. The equality of (98), and (99)), represents the fact that, at $T=T_{c}$, these representations of the spectrum for $T \neq T_{c}$ must give the same result. In the language of perturbed quantum field theory this characterization of the spectrum corresponds to perturbing the $M(3,4)$ minimal model by the energy operator.

There is, however, another Fermionic representation of these same characters which was first conjectured in (2.18) of [40] (and proven in [43])

$$
\chi_{1,1}^{(3,4)}=\sum_{m_{1}=0}^{\infty} \cdots \sum_{m_{8}=0}^{\infty} \frac{q^{\mathbf{m} C_{E_{8}}^{-1} \mathbf{m}^{t}}}{(q)_{m_{1}} \cdots(q)_{m_{8}}},
$$


where $\mathbf{m}=\left(m_{1}, \cdots m_{8}\right)$, and where $C_{E_{8}}$ is the Cartan matrix of the Lie algebra $E_{8}$. Similar identities hold [41] for $\chi_{1,1}^{(3,4)}+\chi_{1,2}^{(3,4)}$ and $\chi_{1,1}^{(3,4)}+\chi_{1,2}^{(3,4)}+\chi_{1,4}^{(3,4)}$. This representation has eight different types of excitations, which have no restriction on the parity of the number of allowed excitations. This is in exact correspondence with the eight particles, found by Zamolodchikov [35] by perturbing the $M(3,4)$ conformal field theory by the spin operator.

For $M(2,5)$ the fermionic forms of the characters have only one quasiparticle, and read

$$
\chi_{1,1}^{(2,5)}=\sum_{n=0}^{\infty} \frac{q^{n^{2}+n}}{(q)_{n}}, \quad \chi_{1,2}^{(2,5)}=\sum_{n=0}^{\infty} \frac{q^{n^{2}}}{(q)_{n}}
$$

which are, in fact, the original identities of Rogers [78] and Ramanujan [79].

\subsection{Scaling theory}

A completely different approach to the Ising model, with $H \neq 0$, is the scaling theory of the renormalization group which was developed before the discovery of conformal and perturbed quantum field theory. This is presented in detail the classic paper of Aharony and Fisher [44, and tested extensively in [1] and [26].

In 444 the conjecture is presented, for $T$ and $H$ real, that

$$
F=-g_{t}^{2} \cdot \ln g_{t}^{2} \cdot \tilde{Y}+g_{t}^{2} \cdot Y_{ \pm}\left(g_{h} /\left|g_{t}\right|^{15 / 8}\right)+A_{0}(t),
$$

where the function $A_{0}(t)$ is analytic at $\tau=0$, and where $Y_{ \pm}(z)$ refer to $T$ above and below $T_{c}$, and

$$
\begin{array}{ll}
g_{t}=\sum_{n=0} a_{2 n}(\tau) \cdot H^{2 n}, & a_{0}(0)=0, \\
g_{h}=\sum_{n=0} b_{2 n+1}(\tau) \cdot H^{2 n+1}, &
\end{array}
$$

with

$$
\begin{aligned}
& a_{2 n}(\tau)=\sum_{j=0}^{\infty} a_{2 n, j} \cdot \tau^{j}, \quad \text { with } \quad a_{0,0}=0, \\
& b_{2 n+1}(\tau)=\sum_{j=0}^{\infty} b_{2 n+1, j} \cdot \tau^{j} .
\end{aligned}
$$

The functions $g_{t}, g_{h}$ may depend on the interaction energies $E(j, k)$ in (11), but the constant $\tilde{Y}$, and the functions $Y_{ \pm}(z)$, do not depend on the interaction energies. The functions $Y_{ \pm}(z)$ are referred to as "scaling functions". The independence on the constants $E(j, k)$ is referred to as "universality". The leading behavior as $T \rightarrow T_{c}$, and $H \rightarrow 0$, of $g_{h} /\left|g_{t}\right|^{15 / 8}$ is:

$$
g_{h} /\left|g_{t}\right|^{15 / 8} \sim \frac{b_{1.0} H}{\left|a_{0,1} \tau\right|^{15 / 8}}=h,
$$


(where the last line is a definition). When $H \neq 0$ the free energy is analytic for all real values of $T$, and, therefore, the functions $Y_{+}(z)$ and $Y_{-}(z)$ must analytically connect with each other as $z \rightarrow \infty$. These are, in fact, parts of the same function. This is conveniently expressed by defining a new variable

$$
\eta=\lim _{H \rightarrow 0, \tau \rightarrow 0} \frac{\tau}{|H|^{8 / 15}} .
$$

Instead (102), we follow [46] in defining the "singular" part of scaling free energy, for $\tau$ and $H$ real, as

$$
F=\frac{\tau^{2}}{8 \pi} \cdot \ln \tau^{2}+|H|^{16 / 15} \cdot \Phi(\eta),
$$

(where we have used the normalization conventions of [46] with the exception that we use $-\tau$ instead of $m$ ). For $H$ and $\tau$ both real and positive, we have

$$
\eta=1 / h^{8 / 15} .
$$

Thus, for real $\eta>0\left(T>T_{c}\right)$, we have

$$
\Phi(\eta)=\eta^{2} \cdot Y_{+}\left(1 / \eta^{15 / 8}\right) .
$$

For $H>0$ and $\tau<0\left(T<T_{c}\right)$, we have

$$
\eta=-1 / h^{15 / 8} .
$$

Thus, for real $\eta<0$ we have:

$$
\Phi(\eta)=\eta^{2} \cdot Y_{-}\left(1 /(-\eta)^{15 / 8}\right) .
$$

The function $\Phi(\eta)$ has, for large values of $\eta$ on the real line of [46], the behavior

$$
\begin{array}{ll}
\Phi(\eta)=\eta^{2} \cdot \sum_{k=1}^{\infty} \tilde{G}_{k} \cdot(-\eta)^{-15 k / 8}, & \eta \rightarrow-\infty, \\
\Phi(\eta)=\eta^{2} \cdot \sum_{k=1}^{\infty} G_{k} \cdot \eta^{-30 k / 8}, &
\end{array}
$$

and, for small values of $\eta$ :

$$
\Phi(\eta)=-\frac{\eta^{2}}{8 \pi} \ln \eta^{2}+\sum_{k=0}^{\infty} \Phi_{k} \cdot \eta^{k} .
$$

For real values of $\eta$ the function $\Phi(\eta)$ has been numerically determined 80 ] by Baxter's variational approach, based on the corner transfer matrix [81, 82], as enhanced by an improved iteration scheme 83.

From the form (102) Aharony and Fisher [44] derive an expression for the functions $F_{ \pm}(\tau)$ of (174), which is supposed to have validity, not only in the limit 
$H \rightarrow 0, \tau \rightarrow 0$, but also for $H$ and $\tau$ finite. Unfortunately, the conjectured form (102) fails for several reasons. First of all, the result of [44 has $F_{+}(\tau)=$ $F_{-}(\tau)$, whereas the analysis of [11, and [26], show that $F_{ \pm}(\tau)$ differ, at order $\tau^{6}$, on the square, triangular and hexagonal lattice. In addition, for the square lattice, the term $\tau^{4}$ of 44 disagrees with [1], 26]. Furthermore the conjecture (102) does not account for the term $B(\tau)$ of (74). Clearly a more general conjecture is required.

A more general conjecture, than that of Aharony and Fisher, utilizes the "irrelevant variable" of scaling theory. We follow the notation of [11] and [26]. We write this more general conjecture, for the singular part of the free energy, as

$$
\begin{gathered}
f_{\text {sing }}\left(g_{t}, g_{h},\left\{g_{u_{j}}\right\}\right)=g_{t}^{2} \ln \left|g_{t}\right| \cdot \tilde{Y}_{ \pm}\left(g_{h} /\left|g_{t}\right|^{15 / 8},\left\{g_{u_{j}} /\left|g_{t}\right|^{y_{j} / y_{t}}\right\}\right) \\
+g_{t}^{2} \cdot Y_{ \pm}\left(g_{h} /\left|g_{t}\right|^{15 / 8},\left\{g_{u_{j}} /\left|g_{t}\right|^{y_{j} / y_{t}}\right\}\right)
\end{gathered}
$$

where, now, the coefficients $a_{2 n}(t)$ and $b_{2 n+1}$, in the nonlinear scaling fields in (104), are allowed to depend on a set of variables $u_{j}$, and where

$$
g_{u_{j}}=\sum_{n=0} c_{2 n}^{j}(t, u) \cdot H^{2 n}
$$

are additional "nonlinear scaling fields" associated with the irrelevant operators of scaling theory that have dimensions $y_{j}$. Further definitions of these concepts may be found for example in [45. However, no explicit forms, or conjectures, for these multivariable formulas have ever been given. Moreover there is no prescription given to separate the effects of the "non linear scaling fields" from the irrelevant operators. In addition the higher powers of $\ln \tau$, seen in the susceptibility, are not present in the form (116). Consequently, while the form (116) may be regarded as "conventional", it is descriptive rather than computational. Indeed, in [11, and [26], even though it is introduced, it is never used.

A further difficulty with the "scaling form" (116) is that it does not give an explanation for the higher powers of $\ln |\tau|$ which occur in $B(\tau)$ of (22). At this point the literature is slightly ambiguous. In [11, in footnote 12 , it is stated that the fact that there is a "resonance between the identity, and the energy", will result in higher powers of $\ln \tau$ (much as integer differences of exponents lead to powers of logarithms in Fuchsian differential equations). However, in footnote 5 of [26], it is said that these powers of $\ln |\tau|$ have "not yet been interpreted within the context of scaling theory". There are clearly things left to be understood.

To see what is needed, we generalize our point of view, from the nearest neighbor Ising model (1), to the much more general case (11), which allows for many further neighbor interactions. This more general model is not integrable. Now the internal energy (12) will include the spin correlations of all spins which are connected by non zero interaction energies. Thus, at least for small values of the non planar bonds, it is entirely reasonable to expect that, at $T \rightarrow T_{c}$, the singularity will not be a pure logarithm, as is the case for the nearest neighbor case, but will involve many higher powers of $\ln ^{p}|\tau|$, each of which are multiplied 
by some suitably high power of $\tau$. We might speculate, from (22), that this power will be $\tau^{p^{2}}$, but there is no further argument for this, except that it is the result found in (22). Moreover, even in the case of the nearest neighbor interactions (11), these terms, with higher powers of $\ln |\tau|$, should be present. By including such terms we will be able to reproduce the "short range" term $B(\tau)$ in the susceptibility expansion (76).

But the truly serious problem in obtaining a general form of the free energy of the Ising model in a magnetic field, as an expansion for "small $H$ and small $\tau$ ", is that the natural boundary, discussed in section 3 for the susceptibility, forces the expansion (74) to be asymptotic. Therefore, even though the susceptibility is well defined for all values of $\tau$, the expansion (74) cannot be taken as the definition of the susceptibility. This is exactly the same problem which afflicts perturbation expansions in the Feynman diagram expansion of quantum field theories, such as Quantum Electrodynamics [30, 31.

\subsection{Non-integrable perturbations for $H \neq 0, \quad T \neq 0$}

The most ambitious program of studying the Ising model, for $H \neq 0$, is to allow both $T \neq T_{c}$, and $H \neq 0$, at the same time. This will allow the passage, from $T<T_{c}, H=0$ to $T>T_{c}, H=0$, on a path in the real $(H, T)$ plane, as is contemplated in the scenario of 32 . However this more general two variables perturbation does not satisfy the criteria of [35], 36] needed for an integrable perturbation. Furthermore, in this more general case, we cannot treat the region of $H \sim 0$, and $T \sim T_{c}$, in isolation from the Lee-Yang edge for complex $H$. Thus, we need to understand how the $M(3,4)$ conformal field theory flows to the $M(2,5)$ theory under the influence of the nonintegrable perturbation.

To make this precise it is necessary to define a scaling limit in the complex plane. This is very delicate because it says that we are describing the singular behaviour of a function of two complex variables in terms of one complex variable. Such a reduction will require very special circumstances to be valid.

Some of the consequences of the existence of this limit have for the free energy are extensively discussed in the 2003 paper of Fonseca and Zamolodchikov [46], where a scenario is presented which incorporates several assumptions about analytical continuation in $T$ and $H$, as two complex variables. We note, however, that the question of the relation of the natural boundary in the complex $T$ plane to the analyticity properties in the complex $\mathrm{H}$ plane near the Lee-Yang edge remains to be investigated.

\section{Why the Ising model is important}

It is very natural to extend perturbed conformal field theory of the Ising model from the integrable cases of [35], [36], where either $H=0, T \neq T_{c}$, and $T=T_{c}, H \neq 0$, to the general case $T \neq T_{c}, H \neq 0$. This has been done in [46]. However, in spite of the impressive results of [80] for the free energy, 
which are in correspondence with the picture of [46], there are several very interesting questions which remain to be addressed.

Perhaps the foremost of these questions is what may be a significant difference between integrable and non-integrable systems. We saw, in section 4.4, that the characters of the conformal field theory of the $M(3,4)$ minimal model have three different representations which are in one to one agreement with the spectrum of excitations, in the massive case, away from the critical point. We can thus think of the integrable perturbations as being precisely tuned to these three different bases, in the same way as degenerate perturbation theory picks out a distinguished basis.

However, in the non-integrable cases, the spectrum of excitations contains a number of particles which depends on both $T$ and $H$, and is not just either one or eight. The implication of this mismatch between the variable number of excitations, seen in the massive model (in what can be considered the infrared part of the spectrum), and the existence of only three particle-like representations of the character in the conformal field theory (which can be considered as the ultraviolet part of the spectrum), is that the smooth match, found in the Ising model at $H=0$ (which was shown in section 3.8 with the precise demonstration by Tracy [68] of the equality (88)), may not hold in the general non-integrable case.

The discussion of the potential disagreement of the short distance behavior of the scaled two-point correlation function for general values of $T$ and $H$ of the Ising model, with the result expected from conformal field theory, raises the suggestion that our understanding of scaling theory may not be complete.

Scaling theory is concerned with the relation of two length scales: the scale of the lattice length on which the theory is defined, and the correlation length which is observed in the correlation functions. In scaling theory, as used in statistical mechanics, and in renormalization of quantum field theory, there is a smooth match between these two scales. But this is drastically different from what occurs in systems, such as fluid mechanics, where a common piece of folk wisdom of perturbation theory [84] is that "divergence should be expected when the solution depends on two independent length scales". This would also seem to be related to the fact, found in [11] and [26], discussed in section 3.8, that the susceptibility does not have a convergent expansion about $T=T_{c}$. It would also seem to be in agreement with the fact that the natural boundary of the susceptibility, presented in section 3.7, does not naturally fit into conventional scaling theory. Thus it may be the case that the smooth matching of long and short distance expansions of the Ising model, at $H=0$, is caused by the integrability of the model, and may not hold in the general non-integrable case.

For these reasons it can certainly be said that, even though the Ising model is the best understood system in statistical mechanics, there are still many puzzling questions to be investigated, questions which have an importance well beyond the narrow range of just this one model. In fact, it can be argued that many of the nagging questions concerning our understanding of quantum field theory [30], 31] are related to the puzzles of the Ising model. Hopefully these questions will inspire future research. 


\section{Acknowledgments}

It is a great pleasure to thank J.H.H. Perk for many insightful discussions and S. Hassani for unpublished results. We also thank C. M. Bender, A. J. Guttmann, B. Nickel, C. A. Tracy, F. Y. Wu for useful comments. One of us (BMM) is grateful to T. Deguchi for the opportunity of presenting a preliminary version this review at the "Tokyo Mathematical Physics Seminar" in July 2011.

\section{References}

[1] L. Onsager, Crystal statistics I. A two dimensional model with an order disorder transition, Phys. Rev. 65 (1944) 117-149.

[2] L. Onsager, discussion, Nuovo Cimento 6 Suppl. (1949) 261.

[3] C.N. Yang, The spontaneous magnetization of the two dimensional Ising model, Phys. Rev. 85 (1952) 808-816.

[4] B. Kaufman and L. Onsager, Crystal statistics III. Short range order in a binary Ising lattice, Phys. Rev. (1949) 1244-1252.

[5] T.T. Wu, Theory of Toeplitz determinants and the spin correlations of the two dimensional Ising model I, Phys. Rev. 149 (1966) 380-401.

[6] T.T. Wu, B.M. McCoy, C.A. Tracy and E. Barouch, Spin-spin correlation functions for the two dimensional Ising model: exact theory in the scaling region, Phys. Rev. B 13 (1976) 315-374.

[7] B.M. McCoy, C.A. Tracy and T.T.Wu, Painlevé equations of the third kind, J. Math. Phys. 18 (1977) 1058-1092.

[8] M. Jimbo and T. Miwa, Studies on holonomic quantum fields XVII, Proc. Jpn. Acad. 56A (1980) 405; and 57A (1981) 347.

[9] B. Nickel, On the singularity of the 2D Ising model susceptibility, J. Phys. A 32 (1999) 3889-3906.

[10] B. Nickel, Addendum to "On the singularity of the 2D Ising model susceptibility", J. Phys. A 33 (2000) 1693-1711.

[11] W. P, Orrick, B.G. Nickel, A.J. Guttmann and J.H.H Perk, The susceptibility of the square lattice Ising model: New developments, J. Stat. Phys. 102 (2001) 795-841.

[12] N. Zenine, S. Boukraa, S. Hassani, J-M. Maillard, The Fuchsian differential equation of the square lattice Ising $\chi^{(3)}$ susceptibility, J. Phys. A: Math. Gen. 37 (2004) 9651-9668.

[13] N. Zenine, S. Boukraa, S. Hassani, J-M. Maillard, Square lattice Ising model susceptibility: Series expansion method and differential equation for $\chi^{(3)}$, J. Phys. A: Math. Gen. 38 (2005) 1875-1899. 
[14] N. Zenine, S. Boukraa, S. Hassani, J-M. Maillard, Ising model susceptibility; The Fuchsian equation for $\chi^{(4)}$ and its factorization properties, J. Phys. A: Math. Gen. 38 (2005) 4149-4173.

[15] N. Zenine, S. Boukraa, S. Hassani and J-M. Maillard, Square lattice Ising model susceptibility: connection matrices and singular behavior of $\chi^{(3)}$ and $\chi^{(4)}$, J. Phys. A 38 (2005) 9439-9474.

[16] S. Boukraa, S. Hassani, J-M. Maillard, B.M. McCoy, J.-A. Weil and N. Zenine, Painlevé versus Fuchs, J. Phys. A39 (2006) 12245-12264. mathphys/0602010

[17] S. Boukraa. S. Hassani, J.-M. Maillard, B.M. McCoy, W.P. Orrick and N.Zenine, Holonomy of Ising model form factors, J. Phys. A40 (2007) 75112.

[18] I. Lyberg and B.M. McCoy, Form factor expansion of the row and diagonal correlation functions of the two dimensional Ising model, J. Phys. A 40 (2007) 3329-3346.

[19] S. Boukraa, S. Hassani, J-M. Maillard, and N. Zenine, Landau Singularities and singularities of holonomic integrals of the Ising class, J. Phys. A40 (2007) 2583-2614.

[20] S. Boukraa, S. Hassani, J-M. Maillard, and N. Zenine, Singularities of $n$ fold integrals of the Ising class and the theory of elliptic curves, J. Phys. A40 (2007) 11713-11748.

[21] S. Boukraa, A.J.Guttmann, S. Hassani, I. Jensen, J-M. Maillard, B. Nickel and N. Zenine, Experimental mathematics on the magnetic susceptibility of the square lattice Ising model, J. Phys. A41 (2008) 455202 (51pp).

[22] A. Bostan, S. Boukraa, S. Hassani, J-M. Maillard J-A Weil and N. Zenine, Globally nilpotent differential operators and the square Ising model, J. Phys. A42 (2009) 125206 (50pp).

[23] A. Bostan, S. Boukraa, A.J. Guttmann, S. Hassani, I. Jensen, J-M. Maillard and N. Zenine, High order Fuchsian equations for the square lattice Ising model: $\tilde{\chi}^{(5)}$, J. Phys. A42 (2009) 275209 (32pp).

[24] S. Boukraa, S. Hassani, I. Jensen. J-M. Maillard, and N. Zenine, High order Fuchsian equations for the square lattice Ising model: $\tilde{\chi}^{(6)}$, J. Phys. A43 (2010) 115201 (22pp).

[25] B. Nickel. I. Jensen, S. Boukraa, A.J. Guttmann, S. Hassani, J-M Maillard and N. Zenine, Square lattice Ising model $\tilde{\chi}^{(5)}$ ODE in exact arithmetic, J. Phys.A 43 (2010) 195205 (24pp).

[26] Y. Chan, A.J. Guttmann, B.G. Nickel and J.H.H. Perk, The Ising susceptibility scaling function, J. Stat. Phy. 145 (2011) 549-590. 
[27] M. Assis, S. Boukraa, S. Hassani, M. van Hoeij, J-M. Maillard and B.M. McCoy, Diagonal Ising susceptibility, elliptic integrals, modular forms and Calabi-Yau equations, J. Phys. A45 (2012) 075205 (32pp).

[28] M. Kashiwara and T. Kawai, Holonomic systems of linear differential equations and Feynman integrals, Publ. RIMS, Kyoto Univ. 12 suppl. (1977) 131-140.

[29] I. Enting and A. J. Guttmann, Solvability of some statistical mechanical systems, Phys. Rev. Lett 76, (1996) 344-347

[30] F.J. Dyson, Divergence of perturbation theory in quantum electrodynamics, Phys. Rev. 85 (1952) 631-632.

[31] G. 't Hooft, Can we make sense out of "Quantum Chromodynamics?", lectures given at the "Ettore Majorana" Int. School of Subnuclear Physics. Erice, July 1977, in "The Why's of Subnuclear Physics" Ed. A. Zichichi, (Plenum, New York/London) 943-971.

[32] B.M. McCoy and T.T. Wu, Two-Dimensional Ising Field Theory in a Magnetic Field: Breakup of the cut in the 2 point function, Phys. Rev. D18, (1978) 1259-1271.

[33] A. Zamolodchikov and I. Ziyatdinov, Inelastic scattering and elastic amplitude in Ising field theory in a weak magnetic field at $T>T_{c}$ : Perturbative analysis, Nucl. Phys. B849 (2011) 654-674.

[34] A. Belavin, A. Polyakov and A.B. Zamolodchikov, Infinite conformal symmetry in two dimensional quantum field theory, Nucl. Phys. B241 (1984) $333-380$.

[35] A.B. Zamolodchikov, Integrable field theory from conformal field theory, Advanced Studies in Pure Mathematics 19 (1989) 641-674.

[36] A.B. Zamolodchikov, Integrals of motion and S-matrix of the (scaled) $T=$ $T_{c}$ Ising model with a magnetic field, Int. J. Mod. Phys. A4 (1989) 42354248 .

[37] J.L. Cardy, Conformal invariance and the Yang-Lee edge singularity in two dimensions, Phys. Rev. Letts. 54 (1985) 1334-1356.

[38] Al.B. Zamolodchikov, Thermodynamic Bethe Ansatz in relativistic models: scaling 3-state Potts and Lee-Yang models, Nucl. Phys. B342 (1990) 695720.

[39] R. Kedem, T.R. Klassen, B.M. McCoy and E. Melzer, Fermionic sum representations for conformal field theory characters, Phys.Letts B 307 (1993) $68-76$ 
[40] R. Kedem. T.R. Klassen, B.M.McCoy and E. Melzer, Fermionic quasiparticle representations for characters of $\frac{\left(G^{(1)}\right)_{1} \times\left(G^{(1)}\right)_{1}}{\left(G^{(2)}\right)_{2}}$, Phys. Letts. B304 (1993) 263-270.

[41] R. Kedem, B.M. McCoy and E. Melzer, The sums of Rogers, Schur and Ramanujan and the Bose-Fermi correspondence in $1+1$ dimensional quantum field theory, in Recent Progress in Statistical Mechanics and Quantum Field Theory, ed. P. Bouwknegt et at. World Scientific (Singapore 1995) 195, hepth 9304056.

[42] A. Berkovich and B.M. McCoy, Rogers-Ramanujan identities: A century of progress from mathematics to physics, Proceedings of ICM98 Documenta Mathematica, (Bielefeld, Germany: Deutscher Mathematiker-Vereinigung) Extra volume ICM 1998, vol. 3 (1998) 163.

[43] S.O. Warnaar and P.A. Pearce, Exceptional structure of the dilute $A_{3}$ model: $E_{8}$ and $E_{7}$ Rogers-Ramanujan identities, J. Phys. A27 (1994) L891897.

[44] A. Aharony and M.E. Fisher, Nonlinear scaling fields and corrections to scaling near critically, Phys. Rev. B 27 (1983) 4394-4400.

[45] M. Caselle, M. Hasenbusch, A. Pelissetto an E. Vicari, Irrelevant operators in the two-dimensional Ising model, J. Phys. A 35 (2002) 4861-4888.

[46] P. Fonseca and A. Zamolodchikov,Ising field theory in a magnetic field: analytic properties of the free energy J. Stat. Phys. 110 (2003) 527-590.

[47] R.J. Baxter, Onsager and Kaufman's calculation of the spontaneous magnetization of the Ising model, J. Stat. Phys. 145 (2011) 518-548.

[48] H. Au-Yang and J.H.H. Perk, Correlation functions and susceptibility in the $z$-invariant Ising model, in: MathPhys Odyssey 2001: Integrable models and beyond, M. Kashiwara and T. Miwa, eds. (Birkäuser, Boston 2002), $23-48$.

[49] E.L. Ince, "Ordinary differential equations". (Dover Publications, New York, 1956).

[50] R. Garnier, Sur des équations différentielles du troisime ordre dont l'intégrale générale est uniforme et sur une classe d'équations nouvelles d'ordre supérieur dont l'intégrale générale a ses points critiques fixes, Ann. Ecole Norm. Sup. 29 (1912) 1-126.

[51] K. Okamoto and H. Kimura, On particular solutions of the Garnier systems and hypergeometric functions of several variables, Quart. J. Math. Oxford (2) 37 (1986) 61-80. 
[52] N.S. Witte, Isomonodromic deformation theory and the next-to-diagonal correlations of the anisotropic square lattice Ising model, J. Phys. A40 (2007) F491-F501.

[53] B.M. McCoy and T.T. Wu, Nonlinear partial difference equations for the two-dimensional Ising model, Phys. Rev. Lett. 45 (1980) 675-678.

[54] J.H.H. Perk, Quadratic identities for Ising model correlations, Phys. Lett.A 79 (1980) 3-5.

[55] A. Zabrodin, Discrete Hirota's equation in quantum integrable models, Int. Journ. of Mod. Phys. B 11, (1997) pp. 3125-3158 and arXiv:hep-th/9610039v1

[56] E. Barouch, B.M. McCoy and T.T. Wu, Zero-field susceptibility of the two dimensional Ising model near $T_{c}$, Phys. Rev. Letts. 31 (1973) 1409-1411.

[57] D.H. Bailey, J.M. Borwein and R.E. Crandall, Integrals of the Ising class, J. Phys. A39 (2006) 12271-12302.

[58] C.A. Tracy, Painlevé transcendents and scaling functions of the two dimensional Ising model, in Nonlinear Equations in Physics and Mathematics, ed. A.O. Barut, D. Riedel Publ. Co., Dortrecht, Holland, (1978) 378-380.

[59] S. Boukraa, S. Hassani, J-M. Maillard, B.M. McCoy, J-A. Weil and N. Zenine, The diagonal Ising susceptibility, J. Phys. A40 (2007) 8219-8236.

[60] X-Z. Wang, Re-examination of the Yang-Lee zeros of the anisotropic Ising models on square, triangular and honeycomb lattices, J. Math. Phys 35 (2002) 1885-1893.

[61] S. Hassani, private communication.

[62] J. Breuer and B. Simon, Natural boundaries and spectral theory, Advances in Math. 226 (2011) 4902-4920.

[63] E. Hille, Analytic function theory, Vol. II, (1962) Chelsea Pub. Comp. N.Y.

[64] B. A. Fuks, Introduction to the Theory of Analytic Functions of Several Complex Variables, Translations of mathematical monographs, v. 8. (1965), American Mathematical Society, Providence, R.I.

[65] C.A. Tracy and B.M. McCoy, Neutron scattering and the correlations of the Ising model near $T_{c}$, Phys. Rev. Letts. 31 (1973) 1500-1504.

[66] J.M. Myers, Wave scattering and the geometry of a strip, J. Math. Phys. 6 (1965) 1839-1846.

[67] B.M. McCoy and J.H.H. Perk, Relation of conformal field theory and deformation theory for the Ising model, Nucl. Phys. B 285[Fs19] (1987) 279-294. 
[68] C.A. Tracy, Asymptotics of a $\tau$-function arising in the two-dimensional Ising model, Commun. Math. Phys. 14 (1991) 297-311.

[69] P. Di Francesco P. Mathieu and D. Sénéchal, Conformal Field Theory (Springer 1996).

[70] G. Mussardo, Statistical Field Theory: an introduction to exactly solved models in statistical physics, ( Oxford Graduate Texts, Oxford University Press, 2009)

[71] T.D. Lee and C.N. Yang, Statistical theory of equations of state and phase transitions.II. Lattice gas and Ising model, Phys. Rev. 87 (1952) 410-419,

[72] V. Matveev and R. Shrock, On properties of the Ising model for complex energy/temperature and magnetic field, J. Phys. A41 (2008) 135002 (22pp).

[73] H.J. Brascamp and H. Kunz, Zeroes of the partition function for the Ising model in the complex temperature plane, J. Math. Phys 15 (1974) 65.

[74] M.E. Fisher, Yang-Lee edge singularity and $\phi^{3}$ field theory, Phys. Rev. Lett. 40 (1973) 1610-1613.

[75] S.O. Warnaar, B. Nienhuis and K.A. Seaton, New construction of solvable lattice models including an Ising model in a field, Phys. Rev. Lett. 69 (1992) 710; A critical Ising model in a magnetic field, Int. J. Mod. Phys. B7 (1993) $3727-3736$.

[76] R.J. Baxter, Rogers-Ramanujan identities in the hard hexagon model, J. Stst. Phys. 26 (1981) 427-452.

[77] A. Roccha-Caridi, in Vertex Operators in Mathematics and Physics, ed. J. Lepowsky et al (Springer, Berlin 1985).

[78] L.J. Rogers, Second memoir on the expansion of certain infinite products, Proc. London Math Soc. (series 1) 25 (1894) 318-343.

[79] L.J. Rogers, S. Ramanujan and G.H. Hardy, Proofs of certain identities in combinatory analysis, Proc. Camb. Phil. Soc. 19 (1919) 211-216.

[80] V.V Mangazeev, M. Yu. Dualev, V.V. Bazhanov and M.T. Batchelor, Scaling and universality in the two dimensional Ising model with a magnetic field, Phys. Rev. E 81 (2010) 060103(R).

[81] R.J. Baxter, Dimers on a rectangular lattice, J. Math. Phys. 9 (1968) 650654 .

[82] R.J. Baxter, Variational approximations for square lattice models in statistical mechanics, J. Stat. Phys. 19 (1978) 461-478.

[83] T. Nishino and K. Okunishi, Corner transfer matrix algorithm for classical renormalization group, J. Phys. Soc. Japan 66 (1997) 3040-3047. 
[84] J.P. Boyd, The devils invention: asymptotic, superasymptotic and hyperasymptotic series, Acta Applicandae Mathematica, 56 (1999) 1-98. 